\title{
Micropolar hypoplasticity for persistent shear band in heterogeneous granular materials
}

\author{
Jia Lin ${ }^{1}$, Wei Wu' ${ }^{1}$, Ronaldo I. Borja ${ }^{2}$ \\ ${ }^{1}$ Institut für Geotechnik, Universität für Bodenkultur, Feistmantelstraße 4, 1180 Vienna, Austria \\ jia.lin@boku.ac.at \\ ${ }^{2}$ Department of Civil and Environmental Engineering, Stanford University, California 94305, USA
}

\begin{abstract}
Persistent shear bands in heterogeneous materials develop progressively, rather than instantaneously. For a meaningful capture of this deformation pattern, the band must develop naturally and freely up to the peak load and well into the softening regime. We use a new micropolar hypoplastic framework to capture the development and propagation of a persistent shear band in a rectangular specimen of silica-concrete sand with spatially varying density. The constitutive framework includes a characteristic length as a regularization parameter, as well as a couple stress representing the angular momentum generated by particle spins. We use computed tomography (CT) imaging and digital image processing to quantify the spatial density variation of the sand, and a void ratio-dependent constitutive model to capture the effect of variable density. Results of the numerical simulations demonstrate the capability of the proposed micropolar hypoplastic framework to track the development and propagation of a persistent shear band in a heterogeneous sand up to the peak load and well into the softening regime.
\end{abstract}

Keywords: finite elements, heterogeneity, hypoplasticity, micropolar theory, persistent shear band

\section{Introduction}

A persistent shear band is a dominant pattern of localized deformation in granular materials. It may or may not be the first one to emerge, but it is the prevailing pattern that persists in the end. Persistent shear bands are often observed in specimens of sand that would otherwise deform homogeneously, such as a rectangular specimen with frictionless end constraints. But material and/or geometric imperfections naturally trigger inhomogeneous deformation. In the presence of material heterogeneity, such as spatially varying density and/or degree of saturation $[1,5$, $7,8,10,50,51,58,71]$, multiple shear bands could emerge and compete for dominance over other shear bands. Many of them would nucleate and grow, only to die out as other more dominant shear bands emerge. In the end, it is the persistent shear band that subsumes all the other weaker shear bands.

Classic rate-independent plasticity theory is often used to detect the inception of a shear band. The seminal paper by Rudnicki and Rice [49] infers the triggering of shear strain localization from the loss of ellipticity of the momentum balance equation, commonly referred to in the literature as the bifurcation criterion $[6,40]$. Beyond the point of bifurcation, mesh- or grid-based 
solutions are known to exhibit mesh sensitivity that is often dealt with by enriching the kinematical description of the shear band. In the context of the finite element method, commonly used enrichments include those provided by the strong discontinuity technique $[11,12,34,42]$ and the extended finite element method [36-38]. However, whereas these enrichments have been shown to provide an effective form of regularization, they do interfere in the natural development of a persistent shear band. This is because the transitional process of embedding a discontinuity into an initially intact material is facilitated by a slip-weakening law characterized by marked softening $[9,27,31,45]$. The process introduces bias into the solution because it forces the shear band to grow from the very first point that bifurcated. For this reason, it is of interest to investigate higher-order constitutive models that do not require artificial enhancements to see if they can provide a more realistic capture of the development and propagation of a persistent shear band.

Generalized continuum theories have characteristic lengths in the constitutive formulation that can be used to solve size-dependent problems. The most commonly used generalized continuum theories include nonlocal theory, strain gradient theory [24, 41], and micropolar theory. Micropolar theory, also known as Cosserat theory [16, 19-21, 25], is the focus of the present paper. The importance of Cosserat theory was noted in the 1968 Freudenstadt-IUTAMSymposium on the Mechanics of Generalized Continua [32]. The theory is specially suited to modeling the constitutive response of granular materials such as sand, whose micromechanics is significantly influenced by particle rotation and frictional work [59]. Compared to other continuum theories, an advantage of micropolar theory is that it takes into account the independent rotations inside the material, which can be linked to the particle rotations inside the shear band. And as noted in the preceding paragraph, micropolar theory does not require an enhancement of the strong discontinuity or extended finite element type (unlike classic plasticity theory) since it is already regularized, and so it allows free development of the persistent shear band. A novel feature of the present work is the new micropolar hypoplastic formulation that accommodates quantified heterogeneity in the constitutive theory.

Classical continuum methods consider a continuous material to be made up of an assemblage of points that can displace linearly in the direction of the stress. This behavior is described by the Cauchy stress tensor and assumes that a force moves through the material in a vector-like fashion. It neglects couple stresses and does not consider the moments induced by locally varying strain conditions. Such description may not be sufficient for certain physical phenomena. For example, particle rotations and the associated frictional work have been shown to dominate the behavior of granular materials. Therefore, to accurately describe the behavior of granular materials, one needs to deal with not only discrete translation but also discrete rotation. Micropolar hypoplasticity has been used successfully to model many characteristic behaviors of granular materials, including those associated with particle rotation $[43,55]$.

Imperfections, whether they are material or geometric in nature, trigger localized deformation in solids. In granular materials such as sand, material imperfection could arise from spatially varying density, among others. Fortunately, density variation can now be quantified determin- 
istically through high-resolution imaging and digital processing. Experimental methods aimed at quantifying spatial density variations have been employed to improve displacement measurement of grid points (see, e.g., [3, 33]), with the main techniques used being GammaRay $[15,17]$ and X-ray CT $[2,4,18]$. Similar advances have been made in digitally correlating successive images to infer the evolution of a shear band, a technique called Digital Image Correlation (DIC) [14, 46-48]. DIC operates by matching pixel patterns between high resolution digital images, and has been used to quantify volumetric evolutions to critical state in dilative sands, measure thickness and inclination of persistent shear bands, and investigate the uniformity of strains along a persistent shear band.

Borja et al. [7] investigated the impact of spatial density variation on the localization of deformation in symmetrically loaded sand bodies using combined experimental-numerical modeling. In their studies, the spatial variation of density was quantified by X-ray CT imaging, which was used for finite element prediction of the system response. Their numerical model was based on classic elasto-plastic finite element analysis with no enrichment, advancing slightly beyond the initial bifurcation to fully resolve the persistent shear band, but not well into the softening regime to avoid issues with loss of uniqueness and mesh sensitivity of the solution. From the DIC results, they demonstrated the ability of their continuum model to predict the location and orientation of the persistent shear band, but they were unable to follow the evolution of this band into the softening regime.

In this paper, we reanalyze the experimental results presented by Borja et al. [7] using micropolar hypoplasticity and classic finite elements without enrichment. The characteristic length of the constitutive model is inferred from the mean grain size of the sand; the density variation is the same as that used in [7]. Central to the present study are the sensitivity analyses conducted to determine an optimal mesh and an optimal step size for load increment. It must be noted that the mesh cannot be too coarse or it will not resolve the shear band properly, but it cannot be too refined either because the finite element dimensions are bounded from below by the size of the sand grains. Since the constitutive formulation has been regularized uniqueness is guaranteed, and thus the solution could continue well into the softening regime without the need for finite element enrichment.

Throughout this paper vectors and tensors are written in boldface, with indicial and symbolic notations used interchangeably. Single tensor contractions are denoted by one dot, $(\boldsymbol{a} \cdot \boldsymbol{b})_{i k}=$ $a_{i j} b_{j k}$ for any second-order tensors $\boldsymbol{a}$ and $\boldsymbol{b}$; whereas double contraction is denoted by two dots, $\boldsymbol{a}: \boldsymbol{b}=a_{i j} b_{i j}$. Vectors and tensors are juxtaposed simply as $(\boldsymbol{a} \boldsymbol{b})_{i j k l}=a_{i j} b_{k l}$ to form higher-order tensors. 


\section{Strong and weak forms}

We consider a body $\mathscr{B}$ bounded by surface $\partial \mathscr{B}$, and assume Dirichlet and Neumann boundaries $\partial \mathscr{B}_{g}$ and $\partial \mathscr{B}_{h}$, respectively. We also assume the usual boundary decomposition of the form $\partial \mathscr{B}=\overline{\partial \mathscr{B}_{g} \cup \mathscr{B}_{h}}$ and $\varnothing=\partial \mathscr{B}_{g} \cap \partial \mathscr{B}_{h}$, where the overline denotes a closure. For a micropolar continuum, the balance of linear momentum takes the form

$$
\rho \frac{D \boldsymbol{v}}{D t}-\nabla \cdot \boldsymbol{s}-\rho \boldsymbol{g}=\mathbf{0},
$$

where $\rho$ is the mass density, $D / D t$ is the material time derivative, $\boldsymbol{v}$ is the solid velocity, $\boldsymbol{s}$ is the nonsymmetric force stress tensor (the term 'force stress' makes it distinct from the 'couple stress' presented subsequently), $\boldsymbol{g}$ is the gravity acceleration vector, and $\boldsymbol{\nabla}$ is the gradient operator. The relevant essential and natural boundary conditions are

$$
\boldsymbol{v}=\overline{\boldsymbol{v}} \quad \text { on } \quad \partial \mathscr{B}_{g} ; \quad \boldsymbol{n} \cdot \boldsymbol{s}=\overline{\boldsymbol{t}} \quad \text { on } \quad \partial \mathscr{B}_{h},
$$

where $\overline{\boldsymbol{v}}$ and $\overline{\boldsymbol{t}}$ are the prescribed line velocity and force traction vectors, respectively (once again, 'force traction' is differentiated from 'couple traction' presented subsequently). The balance of angular momentum can be written in similar form as

$$
\rho \frac{D(\boldsymbol{J} \cdot \boldsymbol{\omega})}{D t}-\nabla \cdot \boldsymbol{\mu}-\rho \boldsymbol{J} \cdot \boldsymbol{c}-\varepsilon(\boldsymbol{s})=\mathbf{0},
$$

where $\boldsymbol{J}$ is the moment of inertia tensor, $\boldsymbol{\omega}$ is the angular velocity, $\boldsymbol{\mu}$ is the skew-symmetric couple stress tensor, $\boldsymbol{c}$ is the body couple vector, and $\varepsilon$ is a permutation operator giving rise to the expression

$$
\varepsilon(\boldsymbol{s})=\left\{\begin{array}{l}
s_{32}-s_{23} \\
s_{13}-s_{31} \\
s_{21}-s_{12}
\end{array}\right\} .
$$

The essential and natural boundary conditions are

$$
\boldsymbol{\omega}=\overline{\boldsymbol{\omega}} \quad \text { on } \quad \partial \mathscr{B}_{g} ; \quad \boldsymbol{n} \cdot \boldsymbol{\mu}=\overline{\boldsymbol{m}} \quad \text { on } \partial \mathscr{B}_{h},
$$

where $\bar{\omega}$ and $\overline{\boldsymbol{m}}$ are the prescribed angular velocity (spin) and couple traction vectors, respectively.

To develop the weak form of the momentum conservation laws, consider a set of trial functions

$$
\mathscr{S}=\left\{\boldsymbol{v}, \boldsymbol{\omega} \mid v_{i}, \omega_{i} \in H^{1} ; \boldsymbol{v}=\overline{\boldsymbol{v}} \text { and } \boldsymbol{\omega}=\overline{\boldsymbol{\omega}} \text { on } \partial \mathscr{B}_{g}\right\}
$$

and a set of variations

$$
\mathscr{V}=\left\{\boldsymbol{\eta}, \boldsymbol{\vartheta} \mid \eta_{i}, \vartheta_{i} \in H^{1} ; \boldsymbol{\eta}=\mathbf{0} \text { and } \boldsymbol{\vartheta}=\mathbf{0} \text { on } \partial \mathscr{B}_{g}\right\} .
$$

We want to find $\boldsymbol{v}, \boldsymbol{\omega} \in \mathscr{S}$ such that for all $\boldsymbol{\eta}, \boldsymbol{\vartheta} \in \mathscr{V}$,

$$
\int_{\mathscr{B}} \boldsymbol{\eta} \cdot\left(\rho \frac{D \boldsymbol{v}}{D t}-\nabla \cdot \boldsymbol{s}-\rho \boldsymbol{g}\right) \mathrm{d} V=0
$$


and

$$
\int_{\mathscr{B}} \boldsymbol{\vartheta} \cdot\left[\rho \frac{D(\boldsymbol{J} \cdot \boldsymbol{\omega})}{D t}-\boldsymbol{\nabla} \cdot \boldsymbol{\mu}-\rho \boldsymbol{J} \cdot \boldsymbol{c}-\varepsilon(\boldsymbol{s})\right] \mathrm{d} V=0 .
$$

Integrating by parts and using the divergence theorem yields the pair of variational equations

$$
\int_{\mathscr{B}} \boldsymbol{\eta} \cdot \rho \frac{D \boldsymbol{v}}{D t} \mathrm{~d} V+\int_{\mathscr{B}}(\boldsymbol{\nabla} \boldsymbol{\eta})^{\top}: \boldsymbol{s} \mathrm{d} V=\int_{\mathscr{B}} \boldsymbol{\eta} \cdot \rho \boldsymbol{g} \mathrm{d} V+\int_{\partial \mathscr{B}_{h}} \boldsymbol{\eta} \cdot \overline{\boldsymbol{t}} \mathrm{d} A
$$

for linear momentum, and

$$
\begin{aligned}
\int_{\mathscr{B}} \boldsymbol{\vartheta} \cdot \rho \frac{D(\boldsymbol{J} \cdot \boldsymbol{\omega})}{D t} \mathrm{~d} V+\int_{\mathscr{B}}(\boldsymbol{\nabla} \boldsymbol{\vartheta})^{\top}: \boldsymbol{\mu} \mathrm{d} V-\int_{\mathscr{B}} \boldsymbol{\vartheta} \cdot \varepsilon(\boldsymbol{s}) \mathrm{d} V \\
=\int_{\mathscr{B}} \boldsymbol{\vartheta} \cdot \rho \boldsymbol{J} \cdot \boldsymbol{c} \mathrm{d} V+\int_{\partial \mathscr{B}_{h}} \boldsymbol{\vartheta} \cdot \overline{\boldsymbol{m}} \mathrm{d} A
\end{aligned}
$$

for angular momentum, where superscript $\mathrm{T}$ is a transposition operator.

For quasi-static loading the inertia terms drop out, and we are left with the pair of equations

$$
\int_{\mathscr{B}}(\boldsymbol{\nabla} \boldsymbol{\eta})^{\top}: \boldsymbol{s} \mathrm{d} V=\int_{\mathscr{B}} \boldsymbol{\eta} \cdot \rho \boldsymbol{g} \mathrm{d} V+\int_{\partial \mathscr{B}_{h}} \boldsymbol{\eta} \cdot \overline{\boldsymbol{t}} \mathrm{d} A
$$

for linear momentum, and

$$
\int_{\mathscr{B}}(\boldsymbol{\nabla} \boldsymbol{\vartheta})^{\top}: \boldsymbol{\mu} \mathrm{d} V-\int_{\mathscr{B}} \boldsymbol{\vartheta} \cdot \varepsilon(\boldsymbol{s}) \mathrm{d} V=\int_{\mathscr{B}} \boldsymbol{\vartheta} \cdot \rho \boldsymbol{J} \cdot \boldsymbol{c} \mathrm{d} V+\int_{\partial \mathscr{B}_{h}} \boldsymbol{\vartheta} \cdot \overline{\boldsymbol{m}} \mathrm{d} A
$$

for angular momentum.

We now consider a plane strain problem on the plane 12 . Here, the only relevant kinematical variables are the line velocities $v_{1}$ and $v_{2}$, and the angular spin $\omega \equiv \omega_{3}$. Therefore, the generalized trial and weighting functions can be written in vector form as

$$
\boldsymbol{V}=\left\{\begin{array}{c}
v_{1} \\
v_{2} \\
\omega
\end{array}\right\}, \quad \boldsymbol{W}=\left\{\begin{array}{c}
\eta_{1} \\
\eta_{2} \\
\vartheta
\end{array}\right\} .
$$

For completeness, we also write the generalized trial function $\boldsymbol{V}$ in time-integrated form as

$$
\boldsymbol{V}=\left\{\begin{array}{c}
v_{1} \\
v_{2} \\
\omega
\end{array}\right\} \quad \Longrightarrow \quad \boldsymbol{U}=\left\{\begin{array}{c}
u_{1} \\
u_{2} \\
\theta
\end{array}\right\}
$$

where $u_{1}$ and $u_{2}$ are the displacement components, and $\theta$ is the rotation. The generalized body and traction vectors can also written as

$$
\boldsymbol{F}=\left\{\begin{array}{c}
\rho g_{1} \\
\rho g_{2} \\
\rho J_{c}
\end{array}\right\}, \quad \boldsymbol{T}=\left\{\begin{array}{c}
\bar{t}_{1} \\
\bar{t}_{2} \\
\bar{m}
\end{array}\right\} ;
$$


whereas the generalized gradient of the weighting functions, as well as the force stresses, are written in the column form

$$
\boldsymbol{G}=\left\{\begin{array}{c}
\eta_{1,1} \\
\eta_{2,2} \\
\eta_{3,3} \\
\eta_{1,2}+\vartheta \\
\eta_{2,1}-\vartheta \\
\vartheta_{, 1} \\
\vartheta_{, 2}
\end{array}\right\}, \quad \boldsymbol{S}=\left\{\begin{array}{c}
s_{11} \\
s_{22} \\
s_{33} \\
s_{12} \\
s_{21} \\
\mu_{31} \\
\mu_{32}
\end{array}\right\}
$$

Therefore, we can write the variational equation for the micropolar theory in the more compact form

$$
\int_{\mathscr{B}} \boldsymbol{G} \cdot \boldsymbol{S} \mathrm{d} V=\int_{\mathscr{B}} \boldsymbol{W} \cdot \boldsymbol{F} \mathrm{d} V+\int_{\partial_{\mathscr{B}_{h}}} \boldsymbol{W} \cdot \boldsymbol{T} \mathrm{d} A .
$$

Similar expressions for the 3D case may be obtained by redefining the generalized vectors.

\section{Finite element formulation}

The finite element formulation for the micropolar continuum model follows the standard Galerkin approximation of the weak form. The matrix problem consists of adding rotational degrees of freedom to the usual translational degrees of freedom at the nodes. Figure 1 depicts the setup for a four-node quadrilateral element.

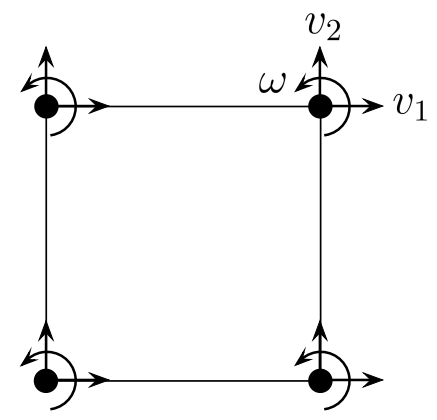

Fig. 1: Standard four-node quadrilateral element enriched with a rotational degree of freedom at each node.

Based on the setup shown in Fig. 1, the strains and curvatures can be evaluated as

$$
\dot{e}=\nabla v+\omega, \quad \dot{\kappa}=\nabla \omega .
$$

We note that an additional term arises from the micropolar spin, $\boldsymbol{\omega}$, which is imposed on top of the usual velocity gradient, $\boldsymbol{\nabla v}$. Now, for 2D plane strain problems on the plane 12 , the 
generalized strain rate vector has a structure similar to that shown in (17), and is written as

$$
\dot{\boldsymbol{E}}=\left\{\begin{array}{c}
v_{1,1} \\
v_{2,2} \\
v_{3,3} \\
v_{1,2}+\omega \\
v_{2,1}-\omega \\
\omega_{, 1} \\
\omega_{, 2}
\end{array}\right\}
$$

The similar structures of the generalized virtual strain vector $G$ and the generalized strain rate vector $\dot{\boldsymbol{E}}$ leads to the standard Galerkin formulation of the finite element problem.

Consider the following finite element interpolation of the trial function within the finite element domain $\mathscr{B}^{e}$

$$
\left.\boldsymbol{V}\right|_{\mathscr{B}^{e}}=\boldsymbol{N}^{e} \dot{\boldsymbol{d}}^{e}, \quad \dot{\boldsymbol{E}}=\boldsymbol{B}^{e} \dot{\boldsymbol{d}}^{e},
$$

and the finite element interpolation of the weighting function

$$
\left.\boldsymbol{W}\right|_{\mathscr{B}^{e}}=\boldsymbol{N}^{e} \boldsymbol{c}^{e},\left.\quad \boldsymbol{G}\right|_{\mathscr{B}^{e}}=\boldsymbol{B}^{e} \boldsymbol{c}^{e} .
$$

In the preceding equations, $\boldsymbol{N}^{e}$ is the element shape function matrix of the form

$$
\boldsymbol{N}^{e}=\left[\boldsymbol{N}_{1}, \boldsymbol{N}_{2}, \ldots, \boldsymbol{N}_{n_{e n}}\right], \quad \boldsymbol{N}_{a}=N_{a}\left[\begin{array}{lll}
1 & 0 & 0 \\
0 & 1 & 0 \\
0 & 0 & 1
\end{array}\right]
$$

whereas $\boldsymbol{B}^{e}$ is a gradient operator that has the following form for 2D plane strain problems

$$
\boldsymbol{B}^{e}=\left[\boldsymbol{B}_{1}, \boldsymbol{B}_{2}, \ldots, \boldsymbol{B}_{n_{e n}}\right], \quad \boldsymbol{B}_{a}=\left[\begin{array}{ccc}
N_{a, 1} & 0 & 0 \\
0 & N_{a, 2} & 0 \\
0 & 0 & 0 \\
N_{a, 2} & 0 & N_{a} \\
0 & N_{a, 1} & -N_{a} \\
0 & 0 & N_{a, 1} \\
0 & 0 & N_{a, 2}
\end{array}\right] ;
$$

$N_{a}$ is the local shape function associated with element node $a, \dot{\boldsymbol{d}}^{e}$ is the generalized element nodal velocity vector, $\boldsymbol{c}^{e}$ is a vector of arbitrary constants, and $n_{e n}$ is the number of element nodes.

In terms of the global shape function matrix $\boldsymbol{N}$ and gradient operator $\boldsymbol{B}$, the variational equation (18) becomes

$$
\underbrace{\int_{\mathscr{B}} \boldsymbol{B}^{\top} \boldsymbol{S} \mathrm{d} V}_{\boldsymbol{F}_{I N T}}=\underbrace{\int_{\mathscr{B}} \boldsymbol{N}^{\top} \boldsymbol{F} \mathrm{d} V+\int_{\partial \mathscr{B}_{h}} \boldsymbol{N}^{\top} \boldsymbol{T} \mathrm{d} A}_{\boldsymbol{F}_{E X T}} .
$$


In practice, we evaluate the internal and external nodal force vectors from the element contributions,

$$
\boldsymbol{f}_{I N T}^{e}=\int_{\mathscr{B} e} \boldsymbol{B}^{e \boldsymbol{\top}} \boldsymbol{S} \mathrm{d} V, \quad \boldsymbol{f}_{E X T}^{e}=\int_{\mathscr{B}} \boldsymbol{N}^{e \boldsymbol{\top}} \boldsymbol{F} \mathrm{d} V+\int_{\partial \mathscr{B} h} \boldsymbol{N}^{e \top} \boldsymbol{T} \mathrm{d} A,
$$

and then assemble the contributions as

$$
\boldsymbol{F}_{I N T}=\AA_{e=1}^{n_{e l}} \boldsymbol{f}_{I N T}^{e}, \quad \boldsymbol{F}_{E X T}=\AA_{e=1}^{n_{e l}} \boldsymbol{f}_{E X T}^{e},
$$

where ' $A$ ' denotes an assembly operator, and $n_{e l}$ is the number of elements.

For hypoplastic formulation, it is more convenient to write the finite element equation in rate form,

$$
\boldsymbol{K} \dot{\boldsymbol{d}}=\dot{\boldsymbol{F}}_{E X T},
$$

where $\boldsymbol{K}$ is the tangent stiffness matrix. If we assume a generalized rate-constitutive equation of the form

$$
\dot{\boldsymbol{S}}=\boldsymbol{H} \dot{\boldsymbol{E}}+\boldsymbol{S}_{w},
$$

where $\boldsymbol{S}_{w}$ emanates from rigid-body rotation to satisfy objectivity, then it is easy to show that $\boldsymbol{K}$ can simply be assembled from element stiffness matrices of the form

$$
\boldsymbol{k}^{e}=\int_{\mathscr{B}^{e}} \boldsymbol{B}^{e \top} \boldsymbol{H} \boldsymbol{B}^{e} \mathrm{~d} V \quad \Longrightarrow \quad \boldsymbol{K}=\AA_{e=1}^{n_{e l}} \boldsymbol{k}^{e} .
$$

The next section develops an expression for the tangent matrix $\boldsymbol{H}$ for the proposed hypoplastic constitutive model.

Before proceeding with the development of the constitutive model, we mention that the solution could be afflicted by mesh locking with full numerical integration in the nearly incompressible regime. This is avoided by employing the $B$-bar method in the numerical integration [29]. The idea of $B$-bar method is to split $\boldsymbol{B}_{a}$ additively into volumetric and deviatoric parts, i.e., $\boldsymbol{B}_{a}=\boldsymbol{B}_{a}^{\mathrm{vol}}+\boldsymbol{B}_{a}^{\mathrm{dev}}$, where

$$
\boldsymbol{B}_{a}^{\mathrm{vol}}=\left[\begin{array}{ccc}
N_{a, 1} / 2 & -N_{a, 2} / 2 & 0 \\
-N_{a, 1} / 2 & N_{a, 2} / 2 & 0 \\
0 & 0 & 0 \\
0 & 0 & 0 \\
0 & 0 & 0 \\
0 & 0 & 0 \\
0 & 0 & 0
\end{array}\right], \quad \boldsymbol{B}_{a}^{\mathrm{dev}}=\boldsymbol{B}_{a}-\boldsymbol{B}_{a}^{\mathrm{vol}}
$$

for 2D plane strain problems. For a four-node quadrilateral element, a one-point integration rule is used for $\boldsymbol{B}_{a}^{\mathrm{vol}} \rightarrow \overline{\boldsymbol{B}}_{a}^{\mathrm{vol}}$ and the standard $2 \times 2$ integration rule is employed for $\boldsymbol{B}_{a}^{\mathrm{dev}}$, yielding

$$
\overline{\boldsymbol{B}}_{a}=\overline{\boldsymbol{B}}_{a}^{\mathrm{vol}}+\boldsymbol{B}_{a}^{\mathrm{dev}} .
$$

Huang et al. [30] employed a similar $B$-bar formulation except that they utilized a volumeaverage rather than a one-point (reduced) integration for the volume term. 


\section{Constitutive theory}

Hypoplasticity is a nonlinear rate-form constitutive theory that has long been used for the shear band analysis and other boundary value problems in granular materials $[22,23,28,39,53,56$, $60,68-70]$. In this section, we shall reformulate this constitutive theory to accommodate the additional kinematics arising from the micropolar theory.

We consider the formulation of $\mathrm{Wu}$ and Kolymbas [65] and write the hypoplastic rateconstitutive equation as the sum of linear and nonlinear terms of the strain rate $\dot{e}$,

$$
\stackrel{\circ}{s}=\mathscr{L}(s): \dot{e}-N(s)\|\dot{e}\| .
$$

The terms $\mathscr{L}$ and $N$ denote the linear and nonlinear components, whereas $\stackrel{s}{s}$ is the Jaumann rate of the force stress tensor defined in terms of the time-derivative of the force stress tensor $\dot{s}$ and the vorticity tensor $w$,

$$
\stackrel{\circ}{s}=\dot{s}+s \cdot w-w \cdot s,
$$

where

$$
\boldsymbol{w}:=\frac{1}{2}(\boldsymbol{\nabla} \boldsymbol{v}-\boldsymbol{v} \boldsymbol{\nabla})
$$

Note that the vorticity tensor $\boldsymbol{w}$ may differ from the micropolar spin $\boldsymbol{\omega}$.

A micropolar hypoplastic constitutive theory has been previously proposed to solve boundary value problems with shear bands $[52,54,55]$. In this theory, the couple stress, curvature, and their rates have been obtained by simple analogy with the original stress-strain constitutive relations (see Huang [30] for further discussions). This formulation is ad hoc and lacks a mathematical underpinning. An alternative way of incorporating micropolar terms into the hypoplastic constitutive framework is by the use of complex tensor formulation described by Lin [35]. Our point of departure is the updated hypoplastic model of Lin, which is based on the following constitutive equation [66]

$$
\stackrel{\circ}{s}=C_{1} \operatorname{tr}(\boldsymbol{s}) \dot{\boldsymbol{e}}+C_{2} \operatorname{tr}(\dot{\boldsymbol{e}}) \boldsymbol{s}+C_{3} \frac{\operatorname{tr}(\boldsymbol{s} \cdot \dot{\boldsymbol{e}})}{\operatorname{tr}(\boldsymbol{s})} \boldsymbol{s}+C_{4}\|\dot{\boldsymbol{e}}\|\left(\boldsymbol{s}+\boldsymbol{s}^{\prime}\right),
$$

where $s^{\prime}$ is the deviatoric part of $s$, and $C_{1}, C_{2}, C_{3}$ and $C_{4}$ are material parameters. Complex tensor formulation yields the following micropolar constitutive equations for the force and couple stresses [35]

$$
\stackrel{\circ}{\boldsymbol{s}}=C_{1} \operatorname{tr}(\boldsymbol{s}) \dot{\boldsymbol{e}}+C_{2} \operatorname{tr}(\dot{\boldsymbol{e}}) \boldsymbol{s}+C_{3} \psi \boldsymbol{s}+C_{4} \sqrt{\|\dot{\boldsymbol{e}}\|^{2}+\ell^{2}\|\dot{\boldsymbol{\kappa}}\|^{2}}\left(\boldsymbol{s}+\boldsymbol{s}^{\prime}\right)
$$

and

$$
\stackrel{\circ}{\boldsymbol{\mu}}=C_{1} \ell^{2} \operatorname{tr}(\boldsymbol{s}) \dot{\boldsymbol{\kappa}}+C_{2} \operatorname{tr}(\dot{\boldsymbol{e}}) \boldsymbol{\mu}+C_{3} \psi \boldsymbol{\mu}+2 C_{4} \sqrt{\|\dot{\boldsymbol{e}}\|^{2}+\ell^{2}\|\dot{\boldsymbol{\kappa}}\|^{2}} \boldsymbol{\mu},
$$

where $\dot{\mu}$ is the Jaumann rate of the couple stress tensor (defined in a similar way as that of the Cauchy stress tensor), $\dot{\kappa}=\nabla \omega$ is the curvature rate tensor, and

$$
\psi=\frac{\operatorname{tr}(\boldsymbol{s} \cdot \dot{\boldsymbol{e}})-\operatorname{tr}(\boldsymbol{\mu} \cdot \dot{\boldsymbol{\kappa}})}{\operatorname{tr}(\boldsymbol{s})}, \quad \operatorname{tr}(\boldsymbol{s}) \neq 0
$$


We note that $\dot{\boldsymbol{\kappa}}, \boldsymbol{\mu}$ and $\boldsymbol{\mu}$ are all skew-symmetric tensors, which means that they have three independent components. The material parameters $C_{1}, C_{2}, C_{3}$, and $C_{4}$ are the same as in the standard hypoplastic formulation. The characteristic length $\ell$ is the only additional material parameter in this micropolar hypoplastic framework.

The response of constitutive equation (33) is governed by the interaction between the linear and nonlinear terms. The perturbation can be either on the linear term or on the nonlinear term, which are equivalent, as shown in $[63,67]$. The linear term can be viewed as constructive, whereas the nonlinear term can be considered as destructive. Enhancing the nonlinear term causes the constitutive response to be contractive, resembling loose material behavior; diminishing it causes the constitutive response to be dilatant, resembling dense material behavior. Therefore, we can describe both dense and loose material behavior with the same constitutive equation by enhancing or diminishing the nonlinear part with density. Following the idea proposed in $[63,67]$, we introduce a multiplier $f_{d}$ into the nonlinear term as a perturbation that incorporates the effect of critical state on the constitutive response. The multiplier has the value $f_{d}=1$ at the critical state, greater than 1 for a loose state, and less than 1 for a dense state. This simple perturbation takes the form

$$
\stackrel{\circ}{s}=C_{1} \operatorname{tr}(\boldsymbol{s}) \dot{\boldsymbol{e}}+C_{2} \operatorname{tr}(\dot{\boldsymbol{e}}) \boldsymbol{s}+C_{3} \psi \boldsymbol{s}+C_{4} f_{d} \sqrt{\|\dot{\boldsymbol{e}}\|^{2}+\ell^{2}\|\dot{\boldsymbol{\kappa}}\|^{2}}\left(\boldsymbol{s}+\boldsymbol{s}^{\prime}\right)
$$

and

$$
\stackrel{\circ}{\boldsymbol{\mu}}=C_{1} \ell^{2} \operatorname{tr}(\boldsymbol{s}) \dot{\boldsymbol{\kappa}}+C_{2} \operatorname{tr}(\dot{\boldsymbol{e}}) \boldsymbol{\mu}+C_{3} \psi \boldsymbol{\mu}+2 C_{4} f_{d} \sqrt{\|\dot{\boldsymbol{e}}\|^{2}+\ell^{2}\|\dot{\boldsymbol{\kappa}}\|^{2}} \boldsymbol{\mu},
$$

where $f_{d}$ is a function of density of the material. It is through $f_{d}$ that the model captures the effect of heterogeneous density on the deformation and localization responses of the material.

The function $f_{d}$ has been studied extensively in some early hypoplastic model formulations, see $[63,64,67]$. It has been shown in these formulations that a linear function of $f_{d}$ is able to capture the effect of density change. Hence, we adopt an expression for $f_{d}$ of the form

$$
f_{d}=(1-a) \frac{e-e_{\min }}{e_{c}-e_{\min }}+a,
$$

where $e$ is the current void ratio (volume of void/volume of solid), $e_{c}$ is the critical void ratio, $e_{\min }$ is the minimum void ratio, and $a$ is a constant less than 1 , which corresponds to the material behavior at the densest state. Figure 2 shows a typical variation of $f_{d}$ with $e$. The sensitivity of the constitutive response to the value of the parameter $a$ is investigated further in the numerical simulations section.

We note that $f_{d} \rightarrow 1$ as $e \rightarrow e_{c}$, and $f_{d} \rightarrow a$ as $e \rightarrow e_{\text {min }}$. The evolution of the void ratio follows the evolution of the volumetric strain according to the following relationship

$$
\frac{\dot{e}}{1+e}=\operatorname{tr}(\dot{\boldsymbol{\varepsilon}})
$$


With the additional term $f_{d}$, we ensure that both dense and loose sands can be modeled with only one set of material parameters. The hypoplastic theory presented above has been previously validated against laboratory test results from various element tests, as well as compared with discrete element calculations [35].

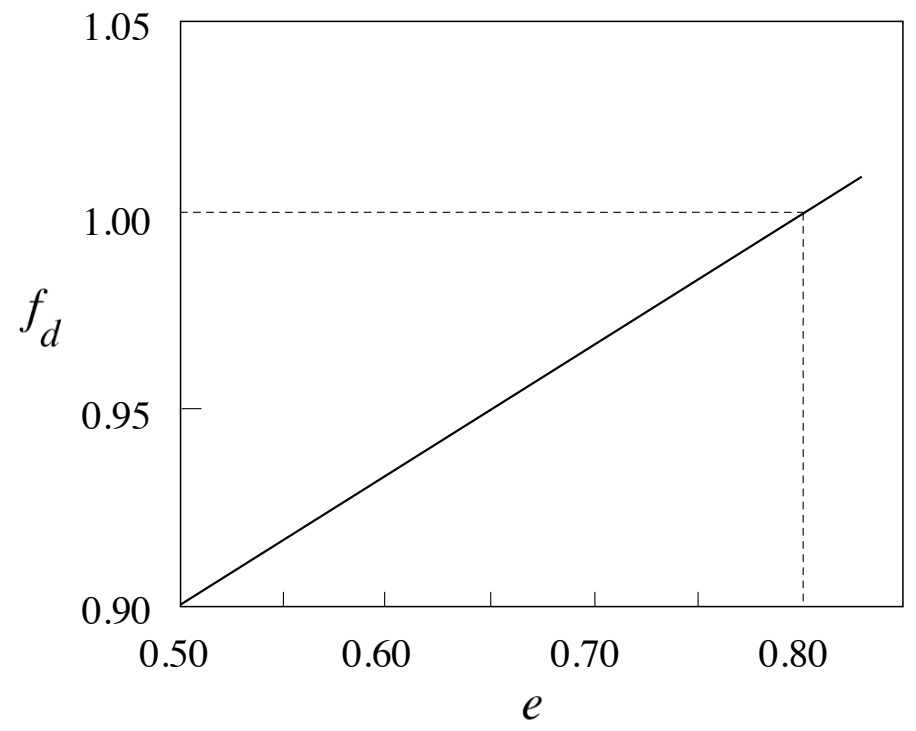

Fig. 2: Relationship between void ratio $e$ and multiplier $f_{d}$.

Because of the highly nonlinear nature of equations (40) and (41), we resort to an explicit numerical integration scheme. In the integration algorithm all stress and state variables are evaluated at time $t_{n}$, so the rate equations effectively take the form

$$
\stackrel{\circ}{\boldsymbol{s}}=C_{1} \operatorname{tr}\left(\boldsymbol{s}_{n}\right) \dot{\boldsymbol{e}}+C_{2} \operatorname{tr}(\dot{\boldsymbol{e}}) \boldsymbol{s}_{n}+C_{3} \psi_{n} \boldsymbol{s}_{n}+C_{4} f_{d n} \sqrt{\|\dot{\boldsymbol{e}}\|^{2}+\ell^{2}\|\dot{\boldsymbol{\kappa}}\|^{2}}\left(\boldsymbol{s}_{n}+\boldsymbol{s}_{n}^{\prime}\right)
$$

and

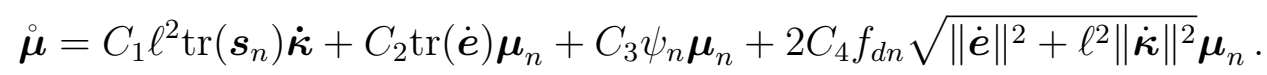

We advance the solution in time by evaluating the tangents (after dropping the time subscript $n$ for brevity)

$$
\begin{aligned}
\frac{\partial \dot{s}_{i j}}{\partial \dot{e}_{m n}} & =C_{1} \Theta I_{i j m n}+C_{2} s_{i j} \delta_{m n}+C_{3} \frac{s_{i j} s_{m n}}{\Theta}+C_{4} f_{d}\left(s_{i j}+s_{i j}^{\prime}\right) \dot{\mathscr{E}}_{m n} \equiv a_{i j m n} \\
\frac{\partial \dot{\mu}_{i j}}{\partial \dot{e}_{m n}} & =C_{2} s_{i j} \delta_{m n}+C_{3} \frac{\mu_{i j} s_{m n}}{\Theta}+2 C_{4} f_{d} \mu_{i j} \dot{\mathscr{E}}_{m n} \equiv b_{i j m n} \\
\frac{\partial \dot{s}_{i j}}{\partial \dot{\kappa}_{m n}} & =-C_{3} \frac{s_{i j} \mu_{m n}}{\Theta}+C_{4} f_{d}\left(s_{i j}+s_{i j}^{\prime}\right) \dot{\mathscr{K}}_{m n} \equiv c_{i j m n} \\
\frac{\partial \dot{\mu}_{i j}}{\partial \dot{\kappa}_{m n}} & =C_{1} \ell^{2} \Theta I_{i j m n}-C_{3} \frac{\mu_{i j} \mu_{m n}}{\Theta}+2 C_{4} f_{d} \mu_{i j} \dot{\mathscr{K}}_{m n}=d_{i j m n}
\end{aligned}
$$

where $I_{i j k l}$ is a rank-four symmetric identity tensor with components

$$
I_{i j m n}=\frac{1}{2}\left(\delta_{i m} \delta_{j n}+\delta_{j m} \delta_{i n}\right)
$$


and

$$
\Theta=\operatorname{tr}(\boldsymbol{s}), \quad \dot{\mathscr{E}}_{m n}=\frac{\dot{e}_{m n}}{\sqrt{\|\dot{\boldsymbol{e}}\|^{2}+\ell^{2}\|\dot{\boldsymbol{\kappa}}\|^{2}}}, \quad \dot{\mathscr{K}}_{m n}=\frac{\ell^{2} \dot{\kappa}_{m n}}{\sqrt{\|\dot{\boldsymbol{e}}\|^{2}+\ell^{2}\|\dot{\boldsymbol{\kappa}}\|^{2}}} .
$$

The constitutive equations can be recast in the following form

$$
\begin{aligned}
& \dot{s}_{i j}=a_{i j m n} \dot{e}_{m n}+b_{i j m n} \dot{\kappa}_{m n}-s_{i k} w_{k j}+w_{i k} s_{k j} \\
& \dot{\mu}_{i j}=c_{i j m n} \dot{e}_{m n}+d_{i j m n} \dot{\kappa}_{m n}-\mu_{i k} w_{k j}+w_{i k} \mu_{k j}
\end{aligned}
$$

For plane strain problems, the tangential tensors may be rearranged in matrix form

$$
\begin{aligned}
a_{i j m n} \rightarrow \boldsymbol{A}_{(5 \times 5)}, & & b_{i j m n} \rightarrow \boldsymbol{B}_{(5 \times 2)} \\
c_{i j m n} \rightarrow \boldsymbol{C}_{(2 \times 5)}, & & d_{i j m n} \rightarrow \boldsymbol{D}_{(2 \times 2)} .
\end{aligned}
$$

Thus, the generalized rate constitutive equation over a finite element domain $\mathscr{B}^{e}$ may be written in matrix form as

$$
\dot{\boldsymbol{S}}=\boldsymbol{H} \dot{\boldsymbol{E}}+\boldsymbol{S}_{w}
$$

where

$$
\boldsymbol{H}=\left[\begin{array}{cc}
\boldsymbol{A} & \boldsymbol{B} \\
\boldsymbol{C} & \boldsymbol{D}
\end{array}\right], \quad \boldsymbol{S}_{w}=\left\{\begin{array}{c}
\boldsymbol{X} \\
\boldsymbol{Y}
\end{array}\right\} .
$$

The vectors $\boldsymbol{X}$ (dimension $5 \times 1$ ) and $\boldsymbol{Y}$ (dimension $2 \times 1$ ) are incremental contributions arising from the rigid-body rotation. For plane strain problem on the plane 12, the vector $\boldsymbol{X}$ can be constructed from the tensor

$$
(-\boldsymbol{s} \cdot \boldsymbol{w}+\boldsymbol{w} \cdot \boldsymbol{s})=w_{12}\left[\begin{array}{ccc}
s_{12}+s_{21} & s_{22}-s_{11} & 0 \\
s_{22}-s_{11} & -s_{12}-s_{21} & 0 \\
0 & 0 & 0
\end{array}\right] \rightarrow \boldsymbol{X}
$$

whereas $\boldsymbol{Y} \equiv \mathbf{0}$.

The constitutive equation can be integrated by assuming a time step $\Delta t$ :

$$
\boldsymbol{S}_{n+1}=\boldsymbol{S}_{n}+\boldsymbol{H} \Delta \boldsymbol{E}+\boldsymbol{S}_{w} \Delta t
$$

where

$$
\left.\Delta \boldsymbol{E}\right|_{\mathscr{B} e}=\boldsymbol{B}^{e} \Delta \boldsymbol{d}^{e}
$$

is the increment of the generalized strain. The solution can be advanced step by step, with all the stress and state variables being updated at each step. The explicit nature of the solution makes it susceptible to numerical instability, and thus, in the next section is mainly devoted to mesh and time-step sensitivity analyses.

\section{Numerical examples}

In this section, we analyze a plane strain compression test with a persistent shear band in a strongly heterogeneous specimen of silica-concrete sand [7]. We also simulate a rigid punch on a heterogeneous medium, similar to the problem of bearing capacity of a strip footing. 


\subsection{Plane strain compression}

Heterogeneity in the sample was measured in terms of density contrast. The specimen was $137 \mathrm{~mm}$ tall, $39.5 \mathrm{~mm}$ wide and $79.7 \mathrm{~mm}$ deep (out-of-plane), with its base resting on a lowfriction, linear bearing sled that permitted lateral offset required for unconstrained shear band propagation. The out-of-plane faces of the specimen were constrained by rigid glass-lined acrylic walls, which enforced zero normal-strain condition and permitted imaging of in-plane specimen deformations. Load cells embedded between the glass and acrylic walls measured the out-of-plane forces. All surfaces contacting the specimen were glass-lined and lubricated to minimize boundary friction.

The sand tested was a $50 \%-50 \%$ by mass sieved mixture of silica and concrete sands. The mixture was relatively uniform, with the median grain diameter of $0.42 \mathrm{~mm}$, coefficient of uniformity of 1.2 , and specific gravity of 2.64 . The specimen was prepared by dry pluviation with the density imperfection imposed by raining from a variable drop height. This resulted in a dense sand specimen with a $3 \mathrm{~cm}$-thick layer of loose sand located below the middle of the specimen as shown in Figure 3(a). After preparation, the sand specimen was confined under $60 \mathrm{kPa}$ vacuum pressure and scanned by X-ray Computed Tomography (CT).

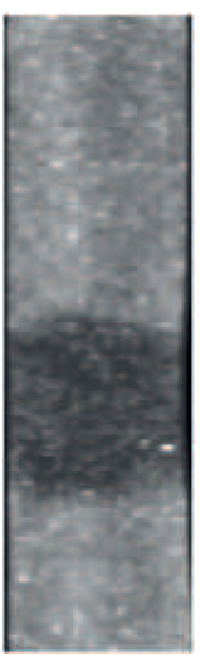

(a)

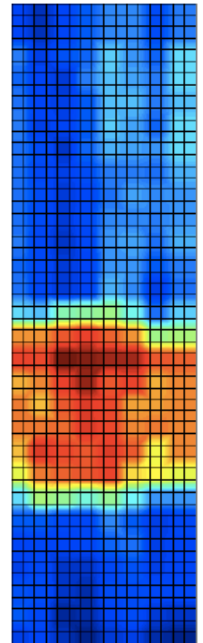

(b)

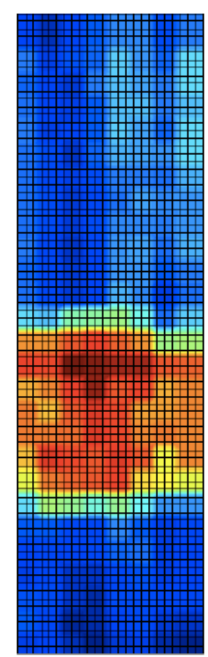

(c)

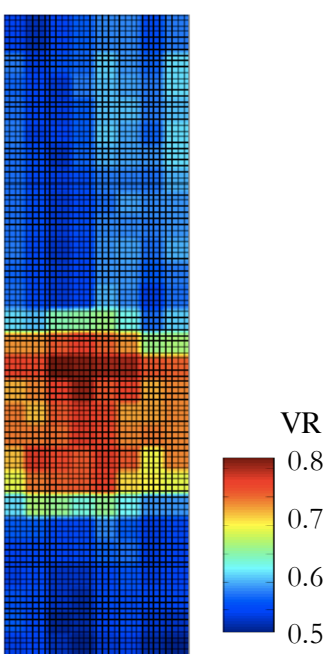

(d)

Fig. 3: Plane strain compression of silica-concrete sand: (a) CT image, (b) Mesh \#1 with 952 nodes and 880 elements, (c) Mesh \#2 with 2075 nodes and 1968 elements, and (d) Mesh \#3 with 3663 nodes and 3520 elements. Color bar is void ratio.

Local material density was calculated from the energy attenuation of an X-ray beam passing through the sand specimen. By collecting attenuation data from multiple directions, local energy attenuations internal to the body, which correlate with local material densities, were back-calculated. Figures 3(b)-(d) show the initial void ratio distribution generated from digital CT-image processing, superimposed with three finite element meshes for sensitivity studies. We remark that progressive refinement was done only on the meshes and not on the description 
of heterogeneity, which was based on the coarsest mesh description, i.e., Mesh \#1. The most refined mesh had element dimensions of about $0.6 \mathrm{~mm}$, which is approximately equal to the mean grain diameter.

After CT scanning, the specimen was placed in the test cell, saturated, consolidated anisotropically to a mean normal effective stress of $130 \mathrm{kPa}$, and then sheared under displacement control. At frequent intervals throughout testing (every $0.1 \%$ axial strain), digital images of in-plane specimen deformations were taken, and digital image correlation (DIC) was performed [47, 48]. Displacement measurements were found to be accurate to within $\pm 0.009 \mathrm{~mm}$. For purposes of analysis, the material parameters were inferred according to the elastic-plastic material model used in [7]. The internal length parameter was taken to be equal to $0.42 \mathrm{~mm}$, which is the mean grain diameter of the sand sample. The hypoplastic material parameter $a$ was taken to be 0.98 (the variation of response with the value of $a$ is investigated further in this section). It has been shown by previous studies $[52,54,55]$ that different initial stochastic distribution of the pore ratio leads to different shear band patterns. Hence, we use the initial void ratio distribution shown in Figure 3 for FE analyses. The remaining material parameters are summarized in Table 1.

\begin{tabular}{cccc}
\hline$E(\mathrm{kPa}) / s_{c}$ & $\phi\left(^{\circ}\right)$ & $\psi\left(^{\circ}\right)$ & $\nu_{i}$ \\
120 & 29 & 0 & 0.2 \\
\hline$C_{1}$ & $C_{2}$ & $C_{3}$ & $C_{4}$ \\
-33.33 & -104.61 & -336.44 & -105.90 \\
\hline
\end{tabular}

Tab. 1: Material parameters for the finite element simulation of plane strain compression test with spatially varying void ratio.

A plane strain test consists of two steps: isotropic loading and vertical compression. For both steps appropriate boundary conditions must be applied. In the following simulations, the bottom nodes were supported on vertical rollers except the middle node, which was pinned to simulate a fully symmetric loading configuration. For isotropic loading the two vertical faces and the top boundary were subjected to a uniform pressure of $112 \mathrm{kPa}$. The micropolar couples on all boundary nodes were set equal to zero. For the hypoplastic model an initial stress was prescribed that balances the isotropic pressure applied on the mesh boundaries during the first step. For the vertical compression step, the top boundary was given a uniform downward displacement while the horizontal displacements were all free. The left and right sides of the sample were free to rotate.

It is generally recognized that micropolar continuum theory results in a numerical solution that depends primarily on an internal length parameter but not on the mesh size. This fact is verified with the three finite element meshes shown in Figure 3. Mesh \#1 contains 952 nodes 


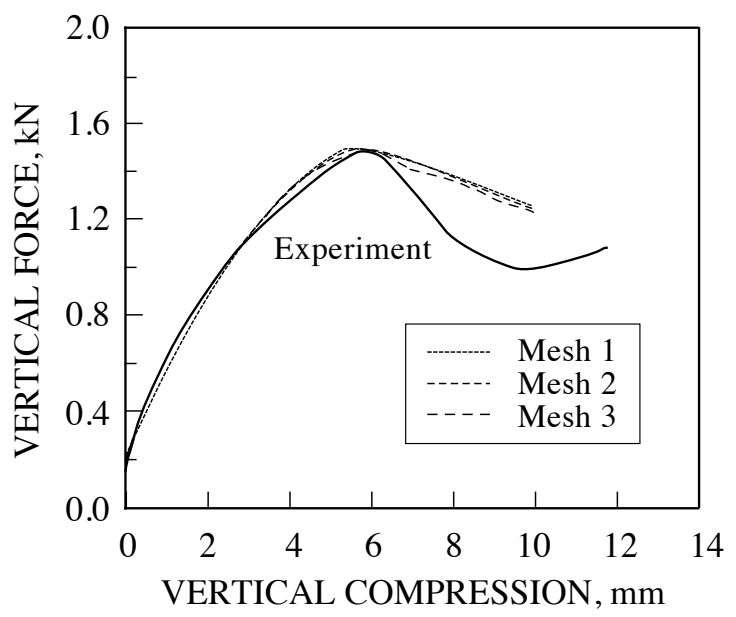

Fig. 4: Vertical force versus vertical compression curve as a function of mesh size.

and 880 elements, Mesh \#2 has 2075 nodes and 1968 elements, and Mesh \#3 has 3663 nodes and 3520 elements. Figure 4 shows that all three meshes resulted in nearly the same loadcompression responses. Note that the curves are nearly identical within the rising part of the response up until the peak load, and that they exhibit very little deviation from each other even in the advanced stage of softening response.

By comparison, the experimental curve shows a rising part followed by softening, after which the curve exhibits a hardening response once again. The hardening response at post-softening is due to the fact that the low-friction linear bearing sled at the bottom of the specimen, which was designed to allow lateral offset for unconstrained shear band propagation, experienced an inadvertent impedance in movement during the course of the experiment [7]. The material parameters of the hypoplastic model are determined according to the initial loading state and critical state. It can be seen in Figure 4 that the initial loading state and the critical state are well predicted by the model while there is an error in predicting the maximum vertical force. If we tune the material parameters to get the same maximum vertical force as in the experiments, the predicted initial strength will be different.

Figures 5, 6, and 7 portray the spatial variation of rotation with mesh size, at vertical compressions of $4.1 \mathrm{~mm}$ (nominal axial strain of 3\%), $5.5 \mathrm{~mm}$ (nominal vertical strain of 4\%) and $8.2 \mathrm{~mm}$ (nominal axial strain of 6\%), respectively. Rotation was calculated as the sum of micropolar rotation and the continuum rotation induced by displacement (counter-clockwise is positive); however, the latter rotation is not so significant compared to the former rotation, and so the rotations plotted in the figures may be considered nearly the same as the micropolar rotations themselves. Note that Mesh \#1 captures all the essential features of the solution, including the unsuccessful development of much weaker conjugate shear bands that seem to cut across the primary shear band during the early stage of loading. Whereas Mesh \#3 appears 
to provide a higher resolution of rotation description than Mesh \#1, it may be considered as "too refined" in this case since the element dimensions are on the order of the mean grain size. Note that the shear bands predicted by the three meshes are not identically located; nevertheless, they are in similar locations and the shear band thicknesses are approximately the same. An interesting conclusion from these figures is that the persistent shear band is not a band in the beginning, but rather, a thick zone of progressively increasing deformation. As the sample is vertically compressed, the deformation gradually concentrates within a narrow zone (see $[13,61,62]$ ), and only when the sample is compressed enough does the true geometric character of the persistent shear band emerge.

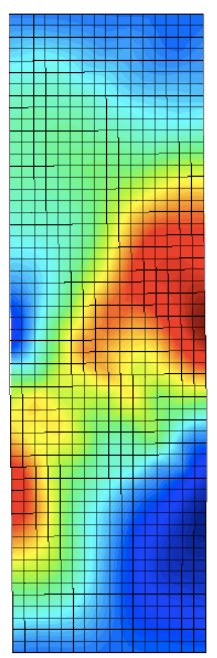

Mesh \#1

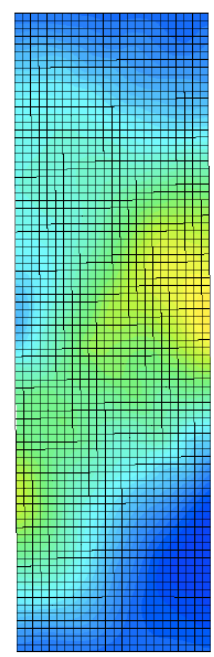

Mesh \#2

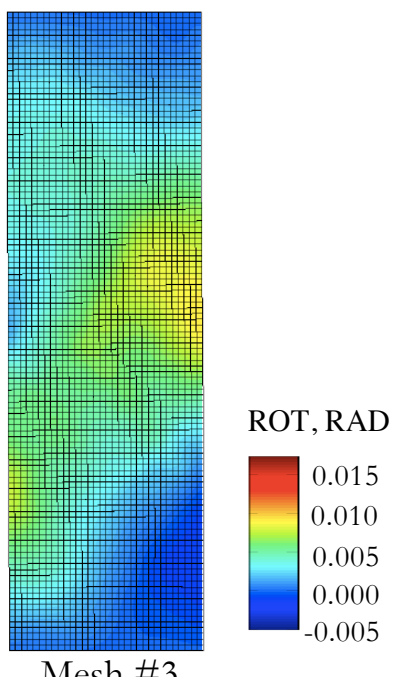

Mesh \#3

Fig. 5: Total rotation in radians as a function of mesh size at a nominal vertical compression of $4.1 \mathrm{~mm}$.

Next we address the issue of time stepping, which is critical for the accuracy of an explicit time integration. To this end, we used Mesh \#1 but with different time steps. Figure 8 compares three load-compression curves showing that the solution is nearly insensitive to the step size. The coarser solution with 40 time steps is comparable in refinement to the solution reported in [7] with conventional elastoplastic theory and a fully implicit backward difference stress-point integration. We have observed from parametric studies that the finer the mesh, the smaller the required time steps. This is necessary in order to capture the variation of deformation occurring across the shear band more accurately.

We next investigate the sensitivity of the solution to the value of the hypoplastic model parameter $a$. To this end, four different values of $a$ were tested: $0.90,0.94,0.98$ and 1.00 . The resulting force versus compression curves are shown in Figure 9. It can be seen that the value of $a$ has a strong influence on the softening behavior of the material. The result with $a=0.90$ seems to be more consistent with the experimental results reported in [7]. Evolution plots of different state variables reported hereafter were generated using the value $a=0.94$. The parameter $a$ has very little effect on the shear band patterns, so they are not shown here. 


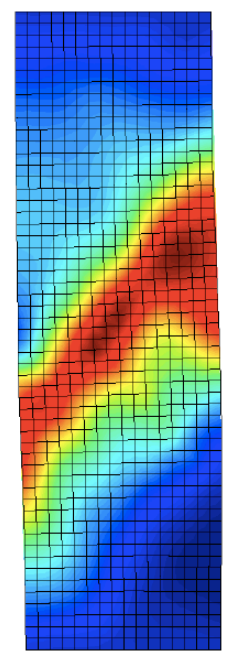

Mesh \#1

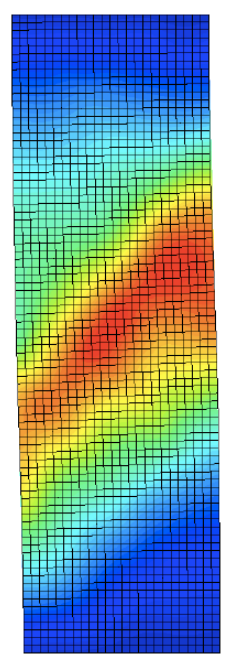

Mesh \#2

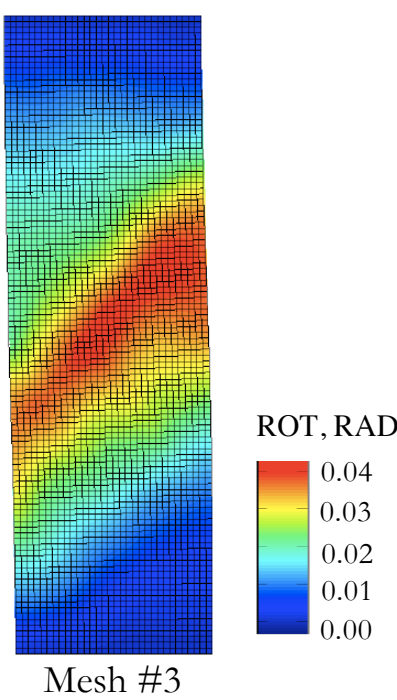

Fig. 6: Total rotation in radians as a function of mesh size at a nominal vertical compression of $5.5 \mathrm{~mm}$.

To better put the numerical simulation results in context, we show in Fig. 10 the quantified shear band measured for the sample using Digital Image Correlation (DIC), see [7]. To generate this image, pixel patterns between high resolution images at vertical compressions of 8 and $8.14 \mathrm{~mm}$ (approximately 6\% nominal vertical strain) were taken, and the incremental displacements were calculated. This results in incremental displacements $u$ and $v$ in the horizontal and vertical directions, respectively. Figure 10 depicts the spatial variation of the norm of these incremental displacements, $\sqrt{u^{2}+v^{2}}$, clearly delineating a distinctive shear band. We note that this snapshot was taken near the lowest point of the softening load-compression branch, where the specimen configuration has not yet been impacted by the inadvertent impedance of the bottom sled.

The same incremental norms are portrayed in Fig. 11 with Mesh \#1. The higher the cumulative nominal vertical strain, the greater is the displacement increment norm and the more pronounced is the shear band. For purposes of comparison, the incremental displacement plot at $6 \%$ cumulative vertical strain in Fig. 11 must be compared with the experimental plot shown in Fig. 10. From these two figures, the calculated shear band appears to be a few degrees flatter and located a little bit higher in the specimen. However, the color bars in the two figures suggest that the calculated displacement increment norms are about the same as those quantified by DIC.

Figure 12 shows the evolution of angular rotation from 3\% vertical strain up until 6\%. Compared with the snapshots shown in Fig. 5 taken at a vertical strain of 2.3\%, the persistent shear band has clearly emerged at $4 \%$ vertical strain with no competition from the other shear bands. Furthermore, the thickness of the shear band appears to be comparable to that of Fig. 10. Note that the rotation also concentrates within the thickness of the shear band. The general sense 


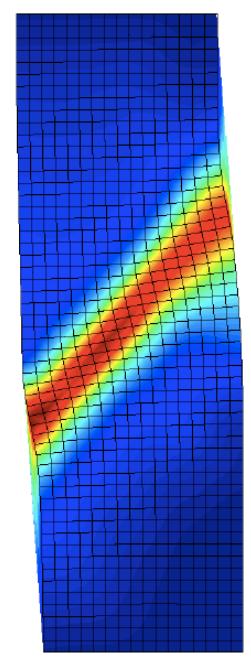

Mesh \#1

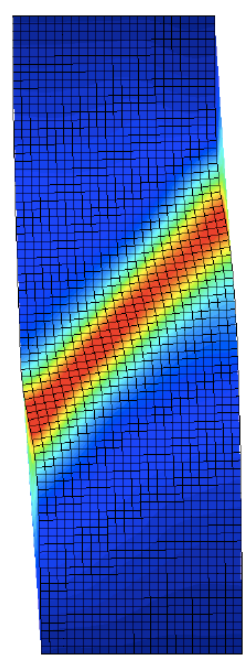

Mesh \#2

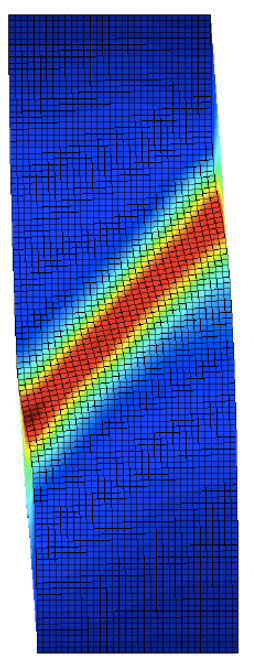

ROT, RAD

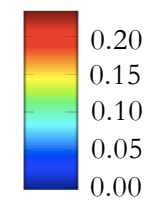

Mesh \#3

Fig. 7: Total rotation in radians as a function of mesh size at a nominal vertical compression of $8.2 \mathrm{~mm}$.

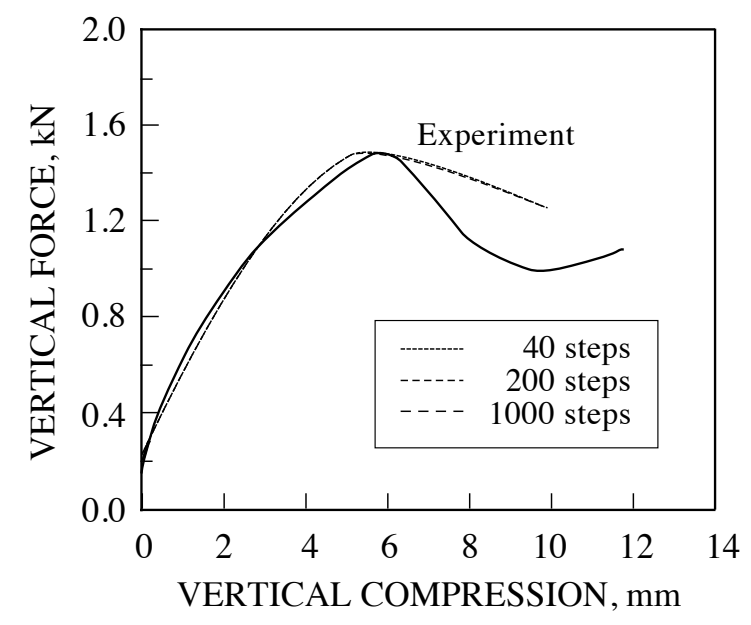

Fig. 8: Vertical force versus vertical compression curve as a function of time step.

of counter-clockwise rotation inside the shear band is consistent with what one would expect from sand particles rolling past each other to realize the prevailing kinematics of the shear band.

Figure 13 suggests that the void ratio distribution has not changed much despite the development of the shear band. Inspection of the numerical results suggests that the void ratio of the loose region decreased and the void ratio of the dense region increased for the elements inside the shear band. It is worthy of note that despite the irregular distribution of initial void 


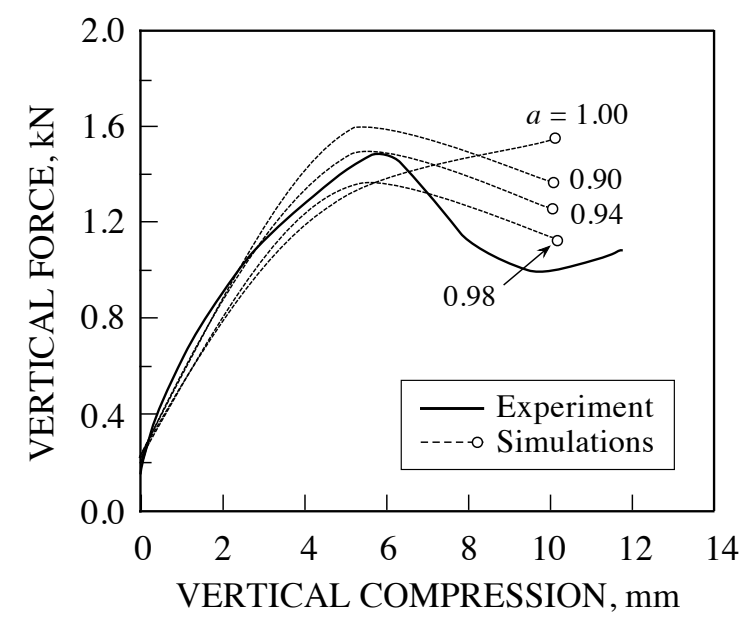

Fig. 9: Vertical force versus vertical compression curve as a function of the hypoplastic constitutive parameter $a$.

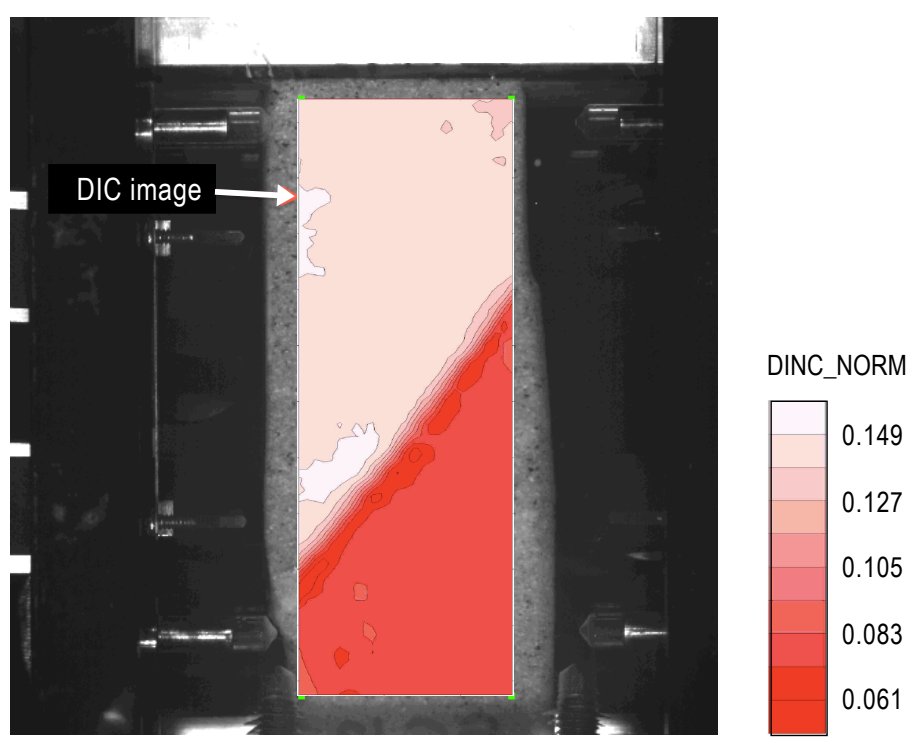

Fig. 10: Norm of incremental displacement $\left(\sqrt{u^{2}+v^{2}}\right)$ from Digital Image Correlation (DIC). Color bar in mm, vertical compression from 8 to $8.14 \mathrm{~mm}$. After Reference [7].

ratio, the predicted trend of the calculated shear band agrees well with that determined from the experiment. We should reemphasize that the numerical model was symmetrically loaded, and that the finite elements had no bias whatsoever. As noted in [7], the imposed heterogeneity in density was strong enough to impact the shear band pattern, irrespective of whether we use classic plasticity or micropolar hypoplasticity. 


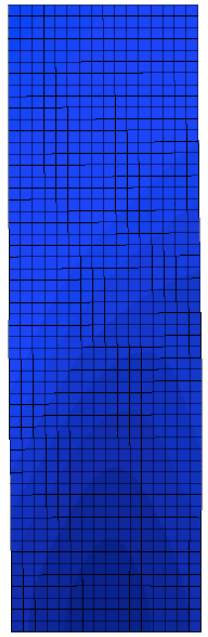

$3 \%$

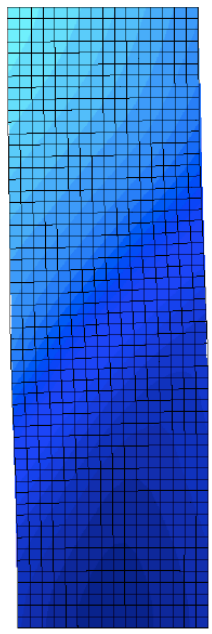

$4 \%$

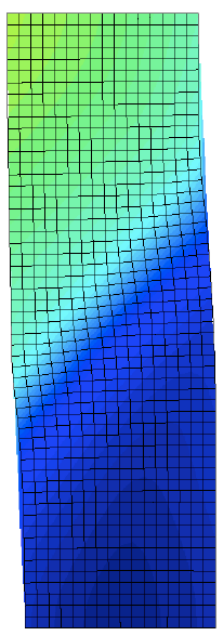

$5 \%$

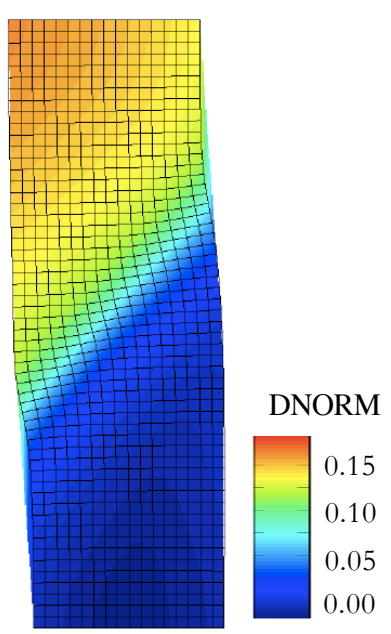

$6 \%$

Fig. 11: Calculated norm of incremental displacement, $\sqrt{u^{2}+v^{2}}$, with Mesh \#1. Numbers in percent are nominal vertical strain. Color bar in $\mathrm{mm}$.

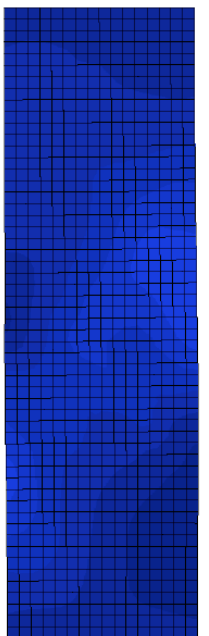

$3 \%$

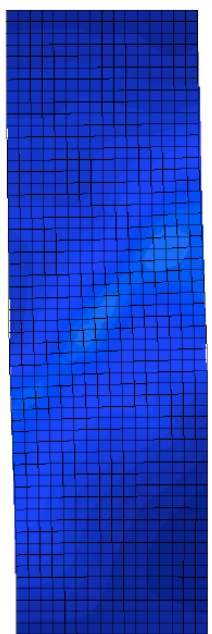

$4 \%$

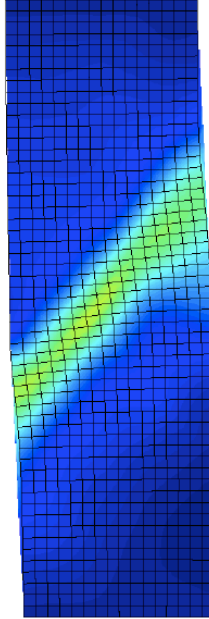

$5 \%$

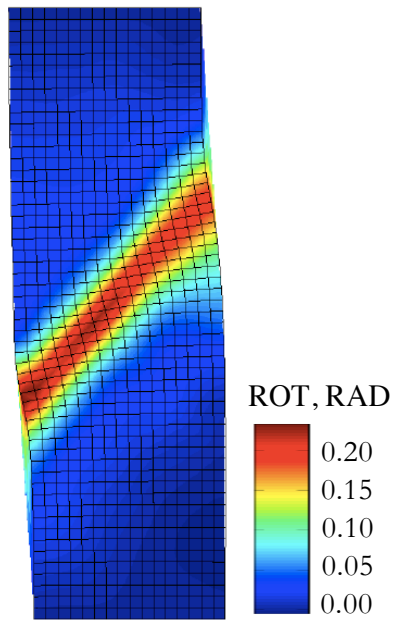

$6 \%$

Fig. 12: Plane strain compression of silica-concrete sand: Numbers in percent are nominal vertical strains. Color bar is rotation in radians, counter-clockwise is positive.

\subsection{Rigid punch problem}

In the previous example, we considered a boundary-value problem in which the material heterogeneity was the only factor that determined the character of the persistent shear band (recall that the boundary constraints in that example were prescribed to favor a homogeneous deformation). In this second example, we consider a boundary-value problem in which both the boundary conditions and imposed material heterogeneity determine the ensuing shear band. 


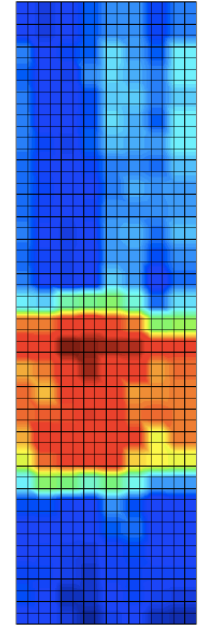

$0 \%$

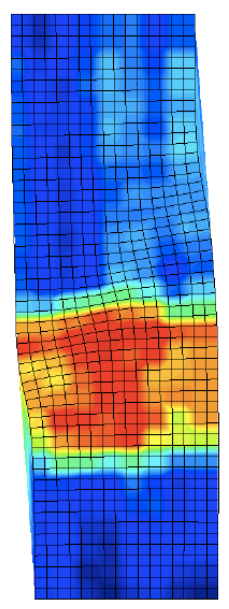

$6 \%$
VOID RATIO

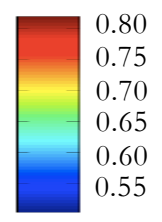

Fig. 13: Plane strain compression of silica-concrete sand before and after vertical compression. Numbers in percent are nominal vertical strain. Color bar is void ratio.

The boundary-value problem consists of a rigid strip footing $2 \mathrm{~m}$ wide pressed into the ground. The foundation domain has a width of $20 \mathrm{~m}$, a depth of $15 \mathrm{~m}$, and the same material properties as in the previous example, except that the internal length parameter was set to $0.1 \mathrm{~m}$ to fit the scale of the problem. The nodes on both vertical sides were supported on horizontal rollers, whereas the bottom nodes were supported on vertical rollers except the middle node, which was pinned to simulate a fully symmetric loading configuration. The footing was modeled as a rigid block able to rotate about the center point, which was given a downward vertical displacement and no horizontal movement. We assumed two cases: Case 1 representing a perfectly homogeneous foundation, and Case 2 representing a heterogeneous foundation. Figure 14 depicts the random void ratio distribution for Case 2 showing a distinct loose region on the right side of the footing. For Case 1, the foundation was assumed to have a uniform void ratio of 0.55 .

Figures 15 and 16 show the deformed meshes and contours of rotation for Cases 1 and 2, respectively. Even though the footing was allowed to rotate, it did not rotate for Case 1 (Fig. 15). Consequently, the localized zone (shear band) is symmetric with respect to the centerline, with the rotation being clockwise on one side and counter-clockwise on the other side. This type of localized deformation was determined by the imposed boundary condition alone. In contrast, the footing rotated in the clockwise direction for Case 2 (Fig. 16) due to the combined effect of boundary constraint and material heterogeneity.

Figure 17 shows the resulting load-displacement curves for Cases 1 and 2. For Case 1 (homogeneous case), a mesh sensitivity analysis was also conducted considering a coarser mesh than the one shown in Fig. 15 (not shown). The two curves (labeled "fine mesh" and "coarse mesh") 


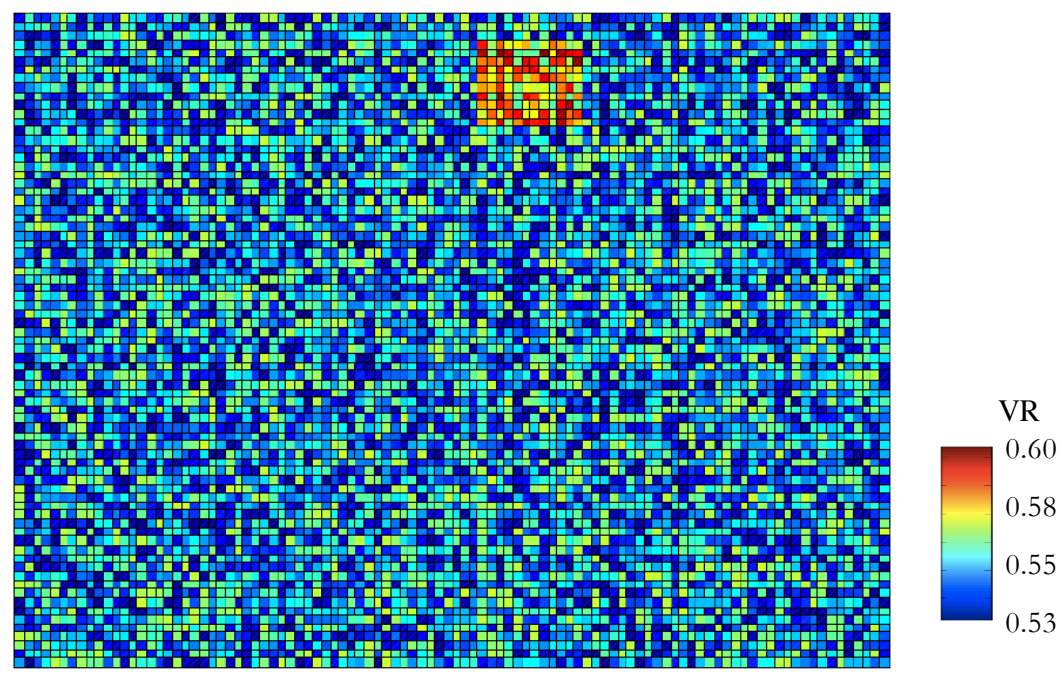

Fig. 14: Void ratio distribution for Case 2 of the rigid punch problem. Color bar is void ratio.

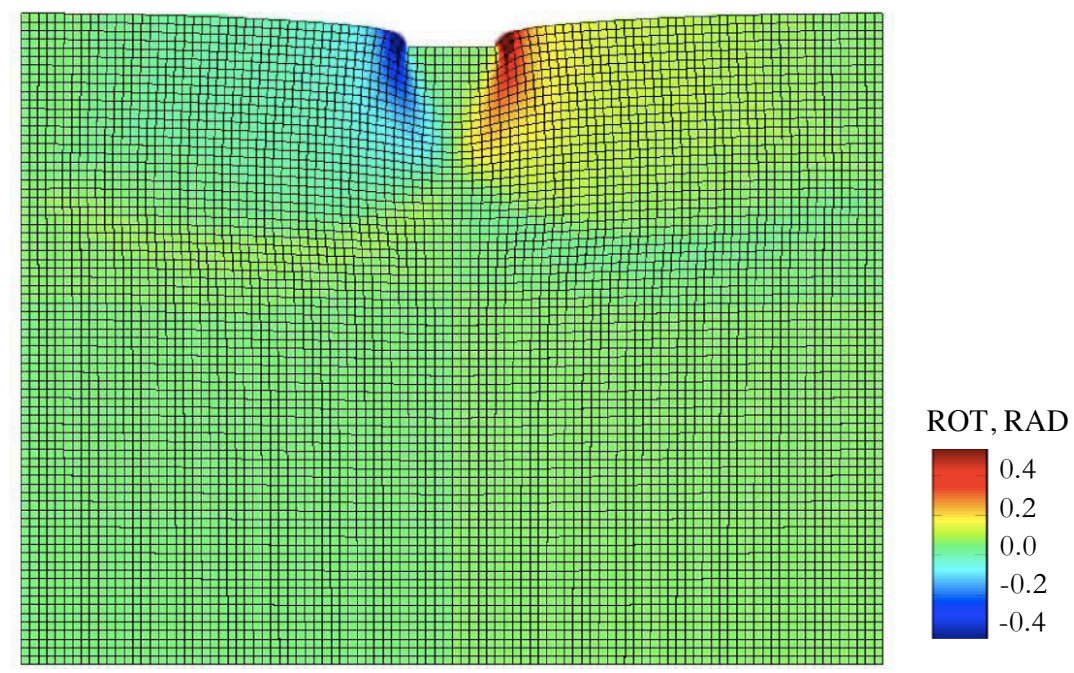

Fig. 15: Deformed mesh and contour of rotation for the rigid punch problem, Case 1. Color bar is rotation in radians, counter-clockwise positive.

show similar load-displacement trends, with the finer mesh exhibiting the expected slightly softer response. This is typical of mesh sensitivity and is not associated with mesh dependency issues. The heterogeneous simulation (Case 2) produced a stronger softening response due to the presence of the loose region, followed by a slightly re-hardening response. The apparent re-hardening could be attributed to the localized zone having developed far enough to reach the left vertical boundary of the mesh, which blocked the shear band from developing further. 


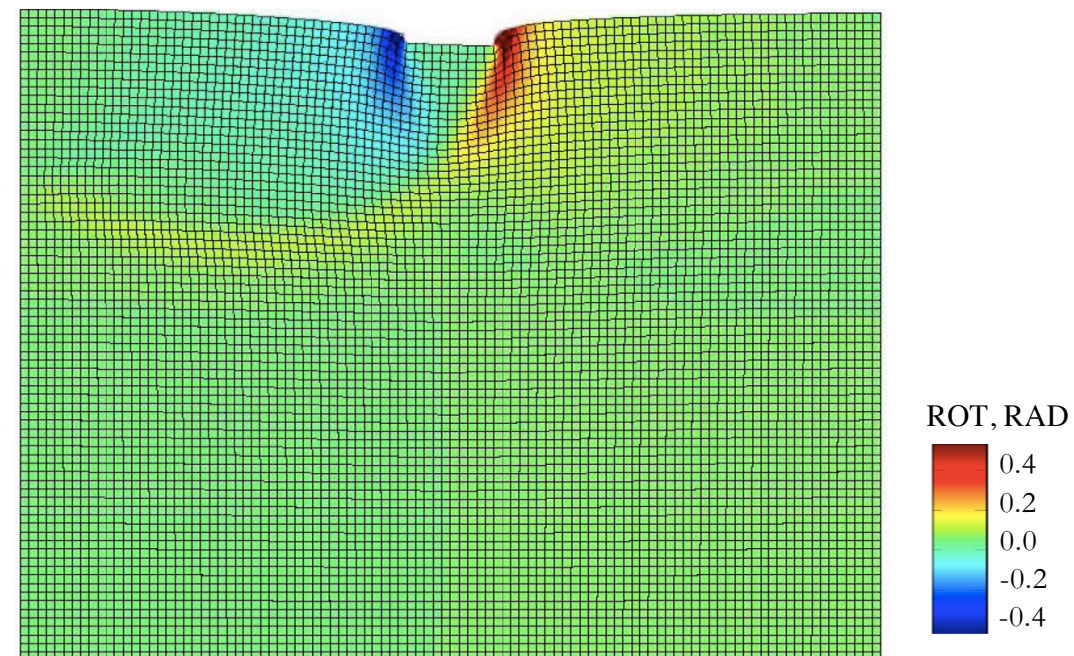

Fig. 16: Deformed mesh and contour of rotation for the rigid punch problem, Case 2. Color bar is rotation in radians, counter-clockwise positive.

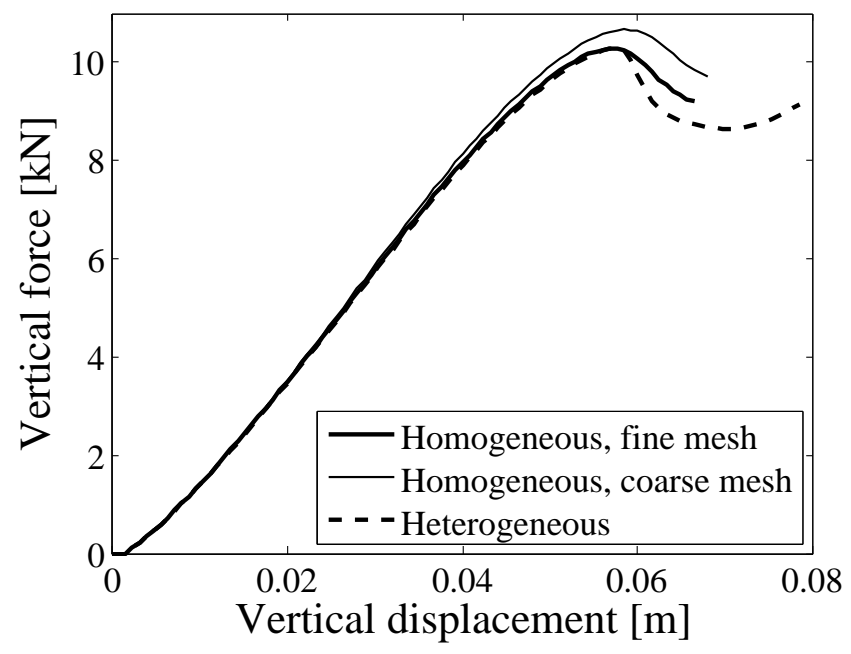

Fig. 17: Load-displacement curves for the rigid punch problem.

\section{Closure}

We have presented a computational framework for capturing the kinematics of a propagating shear band in a strongly heterogeneous granular material. The framework relies on micropolar formulation as a form of regularization, and hypoplasticity to model the heterogeneity in the form of a spatially varying void ratio. The finite element formulation follows the standard Galerkin approximation in which the trial and weighting functions for the force and couple terms were calculated based on the same shape functions. We have used the computational model to reanalyze the development and propagation of a persistent shear band through a 
strongly heterogeneous specimen of silica-concrete sand. We have conducted the simulations well into the softening regime without finite element enrichment, and found the model to be sufficiently predictive, both qualitatively and quantitatively, of the pattern of shear band formation in a heterogeneous sand body. The model was also used to simulate rigid punch of a strip footing on a heterogeneous foundation as a prototype problem where boundary conditions and material heterogeneity determine the character of the ensuing shear band.

Apart from the computational aspects, the most important result of this study is the repeatability of the results with respect to the character of the predicted shear band. It must be emphasized that all of the meshes used in this study were perfectly symmetric. The model had no information whatsoever as to where the persistent shear band would initiate and where it would go. The only information available to the model was the void ratio distribution, yet, irrespective of the mesh the model predicted the same persistent shear band that is consistent with the experiment, see also [7]. Clearly, more experiments of the type considered in this paper are needed to fully validate the predictive capability of the model. But the regularized nature of the constitutive law will surely help simplify any future validation efforts, since it avoids the tricky bifurcation analyses and post-localization enhancements required by classic plasticity formulations.

\section{Acknowledgments}

The third author acknowledges support from the Office of Science, Office of Basic Energy Sciences, of the U.S. Department of Energy under Contract No. DE- FG02-03ER15454 to Stanford University.

\section{References}

[1] R. Alikarami, E. Andó, M. Gkiousas-Kapnisis, A. Torabi, G. Viggiani, Strain localisation and grain breakage in sand under shearing at high mean stress: insights from in situ X-ray tomography, Acta Geotech. 10 (2015) 15-30.

[2] K.A. Alshibli, S. Sture, N.C. Costes, M.L. Frank, F.R. Lankton, S.N. Batiste, R.A. Swanson, Assessment of localized deformations in sand using X-ray computed tomography, Geotechnical Testing Journal 23 (2000) 274-299.

[3] K.A. Alshibli, S. Sture, Shear band formation in plane strain compression, Journal of Geotechnical and Geoenvironmental Engineering 126 (2000) 495-503.

[4] K.A. Alshibli, S. Sture, Sand shear band thickness measurements by digital imaging techniques. Journal of Computing in Civil Engineering 13 (1999) 103-109.

[5] J.E. Andrade, R.I. Borja, Capturing strain localization in dense sands with random density, Int. J. Numer. Meth. Engng. 67 (2006) 1531-1564. 
[6] R.I. Borja, Plasticity Modeling and Computation, Springer-Verlag, Berlin-Heidelberg, 2013.

[7] R.I. Borja, X. Song, A. Rechenmacher, S. Abedi, W. Wu, Shear band in sand with spatially varying density, J. Mech. Phys. Solids 61 (2013) 219-234.

[8] R.I. Borja, X. Song, W. Wu, Critical state plasticity, Part VII: Triggering a shear band in variably saturated porous media, Comput. Methods Appl. Mech. Engrg. 261-262 (2013) 66-82.

[9] R.I. Borja, C.D. Foster, Continuum mathematical modeling of slip weakening in geological systems, J. Geophys. Res. 112(B04301) (2007) doi:10.1029/2005JB004056.

[10] R.I. Borja, J.E. Andrade, Critical state plasticity. Part VI: Meso-scale finite element simulation of strain localization in discrete granular materials, Comput. Methods Appl. Mech. Engrg. 195 (2006) 5115-5140.

[11] R.I. Borja, R.A. Regueiro. Strain localization of frictional materials exhibiting displacement jumps, Comput. Methods Appl. Mech. Engrg. 190 (2001) 2555-2580.

[12] R.I. Borja. A finite element model for strain localization analysis of strongly discontinuous fields based on standard Galerkin approximations, Comput. Methods Appl. Mech. Engrg. 190 (2000) 1529-1549.

[13] G. Buscarnera, Mathematical identification of diffuse and localized instabilities in fluidsaturated sands. Int. J. Numer. Analyt. Meth. Geomech. 38 (2014) 111-141.

[14] O. Chupin, A.L. Rechenmacher, S. Abedi, Finite strain analysis of nonuniform deformation inside shear bands in sands, Int. J. Numer. Analyt. Meth. Geomech. 36 (2012) $1651-1666$.

[15] D.G. Coumoulos, A radiographic study of soils. Ph.D. Dissertation, Cambridge University, Cambridge, U.K., 1968.

[16] E. Cosserat, F. Cosserat, Théorie des Corps Déformables, A. Hermann et Fils, 1909.

[17] J. Desrues, J. Lanier, P. Stutz, Localization of the deformation in tests on sand sample, Engineering Fracture Mechanics 21 (1985) 909-921.

[18] J. Desrues, R. Chambon, M. Mokni, F. Mazerolle, Void ratio evolution Inside shear bands in triaxial sand specimens studied by computed tomography. Géotechnique, 46 (1996) $529-546$.

[19] J. Ericksen, C. Truesdell, Exact theory of stress and strain in rods and shells, Archive for Rational Mechanics and Analysis 1 (1957) 295-323.

[20] A. Eringen, Microcontinuum Field Theories, Springer-Verlag, 2001.

[21] A. Eringen, Continuum Physics: Polar and Non-local Field Theories, Academic Press, 1976. 
[22] C. Fang, W. Wu, On the weak turbulent motions of an isothermal dry granular dense flow with incompressible grains: part II. Complete closure models and numerical simulations, Acta Geotechnica, 9 (2014), 739-752

[23] W. Fuentes, T. Triantafyllidis, A. Lizcano, Hypoplastic model for sands with loading surface, Acta Geotech. 7 (2012) 177-192.

[24] P. Germain, Cours de Mécanique des Milieux Continus. Tome 1.Théorie générale. 1973.

[25] E. Gerolymatou, A micromechanically derived anisotropic micropolar constitutive law for granular media: Elasticity, Int. J. Numer. Analyt. Meth. Geomech. 38 (2014) 17611775 .

[26] P. Guo, Critical length of force chains and shear band thickness in dense granular materials, Acta Geotech. 7 (2012) 41-55.

[27] A. S. Gylland, H. P. Jostad, S. Nordal, Experimental study of strain localization in sensitive clays, Acta Geotech. 2 (2014) 227-240.

[28] J. Hleibieh, D. Wegener, I. Herle, Numerical simulation of a tunnel surrounded by sand under earthquake using a hypoplastic model, Acta Geotechnica, 9 (2014), 631-640

[29] T.J.R. Hughes, Generalization of selective integration procedures to anisotropic and nonlinear media. Int. J. Numer. Meth. Engng. 15 (1980) 1413-1418.

[30] W. Huang, K. Nübel, E. Bauer, Polar extension of a hypoplastic model for granular materials with shear localization, Mechanics of Materials 34 (2002) 563-576.

[31] Y. Ida, Cohesive force across the tip of a longitudinal shear crack and Griffith's specific surface energy, J. Geophys. Res. 77 (1972) 3796-3805.

[32] E. Kröner (Ed.), Mechanics of Generalized Continua: Proceedings of the IUTAMSymposium on the Generalized Cosserat Continuum and the Continuum Theory of Dislocations with Applications, Freudenstadt and Stuttgart (Germany) 1968, Springer-Verlag.

[33] L. Liang, A. Saada, J.L. Figueroa, C.T. Cope, The use of digital image processing in monitoring shear band development. Geotechnical Testing Journal 20 (1997) 324-339.

[34] C. Linder, F. Armero, F., Finite elements with embedded strong discontinuities for the modeling of failure in solids. Int. J. Numer. Meth. Engng. 72 (2007)1391-1433.

[35] J. Lin, Linking DEM with micropolar continuum. Ph.D. Dissertation, Universität für Bodenkultur, Vienna, Austria, 2013.

[36] F. Liu, R. I. Borja, Extended finite element framework for fault rupture dynamics including bulk plasticity. Int. J. Numer. Analyt. Meth. Geomech. 37 (2013) 3087-3111.

[37] F. Liu, R. I. Borja, Stabilized low-order finite elements for frictional contact with the extended finite element method, Comput. Methods Appl. Mech. Engrg. 199 (2010), 24562471. 
[38] F. Liu, R. I. Borja, A contact algorithm for frictional crack propagation with the extended finite element method, Int. J. Num. Meth. Eng. 76 (2008) 1489-1512.

[39] D. Mašín, J. Rott, Small strain stiffness anisotropy of natural sedimentary clays: review and a model, Acta Geotechnica, 9 (2014), 299-312

[40] C. Mihalache, G. Buscarnera, Mathematical identification of diffuse and localized instabilities in fluid-saturated sands. Int. J. Numer. Analyt. Meth. Geomech. 38 (2014) 111-141.

[41] R.D. Mindlin, N.N. Eshel, On first strain-gradient theories in linear elasticity, Int. J. of Solids and Structures 4 (1968) 109-124.

[42] J. Mosler, G. Meschke, 3D modelling of strong discontinuities in elastoplastic solids: fixed and rotating localization formulations. Int. J. Numer. Meth. Engng. 57 (2003) 15531576.

[43] H.-B. Mühlhaus, I. Vardoulakis, Thickness of shear bands in granular materials, Géotechnique 37 (1987) 271-283.

[44] N.-S. Nguyen, H. Magoariec, B. Cambou, Local stress analysis in granular materials at a mesoscale, Int. J. Numer. Analyt. Meth. Geomech. 36 (2012) 1609-1635.

[45] A.C. Palmer, J. R. Rice. The growth of slip surfaces in the progressive failure of overconsolidated clay, Proc. R. Soc. London, Ser. A, 332 (1973) 527-548.

[46] A. L. Rechenmacher, Grain-scale processes governing shear band initiation and evolution in sands, J. Mech. Phys. Solids 54 (2006) 22-45.

[47] A. L. Rechenmacher, S. Abedi, O. Chupin, Evolution of force chains in shear bands in sands, Géotechnique 60 (2011) 343-351.

[48] A.L. Rechenmacher, S. Abedi, O. Chupin, A. Orlando, Characterization of mesoscale instabilities in localized granular shear using digital image correlation, Acta Geotech. 6 (2011) 205-217.

[49] J.W. Rudnicki, J.R. Rice, Conditions for the localization of deformation in pressure sensitive dilatant materials, J. Mech. Phys. Solids 23 (1975) 371-394.

[50] X. Song, R.I. Borja, Mathematical framework for unsaturated flow in the finite deformation range. Int. J. Numer. Meth. Engng. 37 (2014) 658-682.

[51] X. Song, R.I. Borja, Finite deformation and fluid flow in unsaturated soils with random heterogeneity. Vadose Zone J. (2104), doi:10.2136/vzj2013.07.0131.

[52] J. Tejchman, W. Wu, Non-coaxiality and stress-dilatancy rule in granular materials: FE investigation within micro-polar hypoplasticity, International Journal for Numerical and Analytical Methods in Geomechanics (2009), 117-142. 
[53] J. Tejchman, W. Wu, FE-calculations of stress distribution under prismatic and conical sandpiles within hypoplasticity, Granular Matter 10 (2008), 399-405.

[54] J. Tejchman, W. Wu, Dynamic patterning of shear bands in Cosserat continuum, Journal of Engineering Mechanics 123(1997), 123-133.

[55] J. Tejchman, E. Bauer. Numerical simulation of shear band formation with a polar hypoplastic constitutive model, Comput. Geotech. 19 (1996) 221-244.

[56] J. Tejchman, W. Wu, Numerical simulation of shear band formation with a hypoplastic constitutive model, Computers and Geotechnics 18(1996), 71-84.

[57] J. Tejchman, W. Wu, Numerical study on patterning of shear bands in a Cosserat continuum, Acta Mechanica 99 (1993) 61-74.

[58] Z. Tong, P. Fu, S. Zhou, Y. F. Dafalias, Experimental investigation of shear strength of sands with inherent fabric anisotropy, Acta Geotech. 2 (2014) 257-275.

[59] A. Tordesillas, S. Pucilowski, D.M. Walker, J.F. Peters, L.E. Walizer, Micromechanics of vortices in granular media: connection to shear bands and implications for continuum modelling of failure in geomaterials. Int. J. Numer. Analyt. Meth. Geomech. 38 (2014) $247-1275$.

[60] B. Trinh, K. Hackl, Performance of mixed and enhanced finite elements for strain localization in hypoplasticity, Int. J. Numer. Analyt. Meth. Geomech. 36 (2012) 1125-1150.

[61] R. Wan, M. Pinheiro, A. Daouadji, M. Jrad, F. Darve. Diffuse instabilities with transition to localization in loose granular materials. Int. J. Numer. Analyt. Meth. Geomech. 37 (2013) 1292-1311.

[62] G.S. Wang, L.W. Kong, C.F. Wei. An approach to determining the thicknesses of shear bands with an echelon-crack structure. Int. J. Numer. Analyt. Meth. Geomech. 37 (2013) $618-640$.

[63] W. Wu. Hypoplastizität als mathematisches Modell zum mechanischen Verhalten granularer Stoffe, Ph.D. Dissertation, Karlsruhe University, 1992.

[64] W. Wu, E. Bauer, E. Kolymbas, ed., (1993), Modern Approaches to Plasticity, chapter A hypoplastic constitutive model for barotropy and pyknotropy of granular soils, pp. 365383.

[65] W. Wu, D. Kolymbas, Numerical testing of the stability criterion for hypoplastic constitutive equations, Mechanics of Materials 9(1990), 245-253.

[66] W. Wu, E. Bauer, A simple hypoplastic constitutive model for sand, International Journal for Numerical and Analytical Methods in Geomechanics 18(1994), 833-862.

[67] W. Wu, E. Bauer, D. Kolymbas, Hypoplastic constitutive model with critical state for granular materials, Mechanics of Materials 23(1996), 45-69. 
[68] W, Wu, Z. Sikora, Localized bifurcation in hypoplasticity, International Journal of Engineering Science 29 (1991), 195-201.

[69] W. Wu, Non-linear analysis of shear band formation in sand, International Journal for Numerical and Analytical Methods in Geomechanics 24(2000), 245-263.

[70] W. Wu, On high-order hypoplastic models for granular materials, Journal of Engineering Mathematics 56(2006), 23-34.

[71] L. Zhuang, Y. Nakata, U. Kim, D. Kim, Influence of relative density, particle shape, and stress path on the plane strain compression behavior of granular materials, Acta Geotech. 2 (2014) 241-255. 